\title{
Perfil Epidemiológico de Pacientes Notificados com Hanseníase, em uma Cidade do Norte de Minas no Período de 2009-2013
}

\section{Epidemiological Profile of Patients Notified With Leprosy in a City in the North of Minas in the Period of 2009-2013}

\author{
Eugênia Maria Mendes de Aquino ${ }^{1}$ \\ Cleyrianne Alves de Souza ${ }^{1}$ \\ Lorena Aguilar Xavier ${ }^{2}$ \\ Marina Ramos Costa ${ }^{2}$ \\ Jacqueline Teixeira Teles Gonçalves ${ }^{3}$ \\ Karina Andrade de Prince 4 \\ Laércio Fonseca Costa ${ }^{5}$ \\ Luçandra Ramos Espírito Santo ${ }^{6}$
}

\section{RESUMO}

Objetivo: Descrever o perfil epidemiológico dos pacientes notificados com hanseníase no Município de Montes Claros, no período de 2009-2013, disponibilizados no Sistema de Informação de Agravos e Notificação-SINAN. Metodologia: Realizou-se um estudo epidemiológico observacional retrospectivo, que envolveu uma amostra de 225 casos confirmados de hanseníase. Critério de inclusão correspondeu aos hansenianos diagnosticados, residentes em Montes Claros e notificados no Sistema de Informação de Agravos de Notificação (SINAN). Resultados: As variáveis do estudo foram divididas em sete categorias de análise: ano; sexo; faixa etária mais acometida; classificação operacional; forma clínica; avaliação do grau de incapacidade física no diagnóstico e esquema terapêutico. Foram realizadas análises descritivas dos dados, em que se verificou os seguintes dados: No período de 2009-2013 foram notificados 225 novos casos de hanseníase, sendo o ano de 2009 o de maior índice de infecção chegando a 73 casos, correspondendo a $32,44 \%$ destes, acometendo, principalmente, homens (56\%). A faixa etária prevalente foi 20 a 39 anos e 40 a 59 anos. O grau zero de incapacidade foi o mais encontrado $(76 \%)$. A forma clínica prevalente foi a dimorfa $(44,89 \%)$, a classe operacional foi a multibacilar $(87,11 \%)$, com poliquimioterapiamultibacilar $(85,78 \%)$. Conclusão: Após a análise dos dados estudados chegou-se à conclusão que o número de casos de hanseníase diagnosticados em Montes Claros-MG apresentou uma redução. A forma multibacilar ainda foi a predominante. Embora o número de casos de hanseníase tenha evidenciado uma redução, a doença ainda é preocupante.

DESCRITORES: Hanseníase, Mycobacterium leprae, Epidemiologia, Notificação de doenças.

\begin{abstract}
Objective: To describe the epidemiological profile of the patients notified with leprosy in the municipality of Montes Claros, in the period of 2009-2013, available in the SINAN Notification and Injury Information System. Methodology: This was a retrospective observational epidemiological study involving a sample of 225 confirmed cases of leprosy. Inclusion criterion corresponded to the diagnosed hansenians, living in Montes Claros and notified in the Notification of Injury Information System (SINAN). Results: The study variables were divided into seven categories of analysis: year; sex; Age group; Operational classification; Clinical form; Evaluation of the degree of physical incapacity in the diagnosis and therapeutic scheme. Descriptive analysis of the data were carried out, in which the following data were verified: In the 2009-2013 period, 225 new cases of leprosy were reported, 2009 being the one with the highest infection rate, reaching 73 cases corresponding to $32.44 \%$ of these; Affecting mainly men $(56 \%)$. The prevalent age range was 20 to 39 years and 40 to 59 years. The zero degree of disability was mostly found (76\%). The prevalent clinical form was dimorphic $(44.89 \%)$, the operational class was multibacillary $(87.11 \%)$, with polyquimioterapia multibacilar $(85.78 \%)$. Conclusion: After analyzing the data, we conclude that the number of leprosy cases diagnosed in Montes Claros-MG reduced. The multibacillary form is still predominant, although the number of cases of reduced leprosy is still worrying.
\end{abstract}

DESCRIPTORS: Leprosy, Mycobacterium lepra, Epidemiology, Disease Notification.

1 - Graduada em Farmácia pelas Faculdades Unidas do Norte de Minas - FUNORTE. Montes Claros, Minas Gerais, Brasil.

2 - Graduanda do curso Médico da Universidade Estadual de Montes Claros - Unimontes. Montes Claros, Minas Gerais, Brasil.

3 - Mestre em Ciências da Saúde pela Universidade Estadual de Montes Claros - Unimontes. Docente das Faculdades Integradas Pitágoras - FIPMOC, Faculdades Unidas do Norte de Minas - FUNORTE. Montes Claros, Minas Gerais, Brasil.

4 - Doutora em Biociências e biotecnologia aplicadas à farmácia pela Faculdade de Ciências Farmacêuticas UNESP - Araraquara (SP). Docente das Faculdades Integradas Pitágoras - FIPMOC. Montes Claros, Minas Gerais, Brasil.

5 - Docente das Faculdades Integradas Pitágoras - FIPMOC e Faculdades Unidas do Norte de Minas - FUNORTE. Médico do Hospital Universitário Clemente de Faria. Montes Claros, Minas Gerais, Brasil.

6 - Doutoranda em Ciências da Saúde pela Universidade Estadual de Montes Claros - Unimontes. Docente das Faculdades Integradas Pitágoras - FIPMOC, Faculdades Unidas do Norte de Minas - FUNORTE e Universidade Estadual de Montes Claros - UNIMONTES do Departamento de Saúde Mental e Coletiva. Montes Claros, Minas Gerais, Brasil. 
A s referências mais remotas da Hanseníase datam há 600 a.C., onde era considerada uma doença contagiosa, mutilante e incurável, quando atitudes preconceituosas de rejeição e discriminação ao portador, o qual, comumente, era excluído da sociedade ${ }^{1}$.

Causada pelo Mycobacterium Leprae (M.Leprae), a Hanseníase é uma doença infectocontagiosa de evolução crônica, que afeta a pele, nervos periféricos e, ocasionalmente, órgãos internos e mucosas. Os hansenianos são Identificados por lesões cutâneas com diminuição de sensibilidade térmica, tátil e dolorosa, isso devido à predileção M.Leprae em acometer as células cutâneas e nervosas periféricas ${ }^{2}$.

A manifestação da doença é indeterminada, pois a maioria dos indivíduos apresenta respostas imunocelulares ao agente causador da doença de hanseníase, pré-determinadas pela capacidade inata do indivíduo em resistir ou não a infecção².

O diagnóstico precoce da Hanseníase e o seu tratamento adequado evitam a evolução da doença e, consequentemente, impedem a instalação das incapacidades físicas por ela provocadas. A transmissão se dar pelo convívio com os doentes do tipo virchowiano ou dimorfo, os quais não foram diagnosticados e/ou não deram início ao tratamento. As principais vias de transmissão são as mucosas das vias aéreas superiores. O M.Leprae tem capacidade de infectar grande número de pessoas, contudo, não são todos que desenvolvem a doença. O diagnóstico e tratamento da infecção são fáceis e leva a cura em tempo relativamente curto, feito pelo esquema de poliquimeoterapia. Em contra partida ainda é uma preocupação mundial a infecção causada pela hanseníase uma vez que muitos países ainda não conseguiram eliminar essa doença, entre eles o Brasil ${ }^{1}$.

Em 2011, a Secretaria de Vigilância em Saúde criou a Coordenação Geral da Hanseníase e Doenças em Eliminação CGHDE (Decreto $n^{\circ}$ 7.530, de 21 de Julho de 2011), com o objetivo de fortalecer as respostas para um grupo doenças em que os resultados dos programas nacionais foram considerados insuficientes e incompatíveis com a capacidade do SUS de resolução dos problemas de saúde da população ${ }^{3}$.

O Brasil, segundo pesquisa realizada em 2008 , tende a diminuir significativamente a estatística para s séries temporais de coeficientes de detecção. Contudo, em 1990 a 2008 esse coeficiente obteve variáveis entre 20,0/100.000 habitantes em 1990 e em 2013, 29,4/100.000 habitantes, sendo a classificação muito alta, segundo parâmetros oficiais $^{4}$.

Em Minas Gerais, segundo o mesmo estudo, tende a diminuir significativamente e estatisticamente para as series temporais de coeficiente detecção. Contudo, em 1990 a 2008 esse coeficiente obteve variáveis entre 17,55/100.000 habitantes em 1992 e 9,75/100.000 habitantes em 2008, tendo classificação "alta”, de acordo com dados oficiais ${ }^{4}$.

A Região Sudeste, tem coeficiente com valor médio de 13,71/100.000 habitantes, tendo variável 16,16/100.000 em 1997 e 8,81/100.000 em 2008, por isso a classificação alta no período ${ }^{4}$.

A Cidade de Montes Claros no período de 2005 a 2009 obteve um coeficiente médio anual de detecção 1,87/100.000 habitante, resultado elevado quando comparado ao do Estado de Minas Gerais, classificando assim, a cidade como sendo de alto nível endêmico 5 .

Tratando-se de uma Doença de notificação compulsória e investigação obrigatória, depois de concluído o diagnóstico da doença, deverá ser feito juntamente com o órgão de Vigilância Epidemiologia hierarquicamente superior as notificações dos casos, por meio de uma ficha de notificação de investigação do Sistema de Informações do Agravo e Notificação (SINAN) ${ }^{6}$.

Este estudo teve como objetivo analisar epidemiologicamente os portadores da doença de hanseníase no Município de Montes Claros no 
Norte de Minas Gerais, compreendendo o período de 2009 a 2013 e, assim, estabelecer indicadores, onde será possível a análise, a tendência do comportamento da doença, os agravos, a avaliação de incapacidade dos acometidos pela doença, bem como auxiliar os profissionais da área da saúde na prevenção, no tratamento e na cura da doença.

\section{Metodologia}

Realizou-se um estudo epidemiológico observacional retrospectivo, o qual envolveu uma amostra de 225 casos confirmados de hanseníase. Adotou-se como critério de inclusão os hansenianos diagnosticados, residentes em Montes Claros e notificados no Sistema de Informação de Agravos de Notificação (SINAN). Esse sistema é gerenciado pelo Sistema Nacional da Fundação Nacional de Saúde, que é o órgão responsável pela elaboração e expedição de normas que regem sua utilização e operacionalização em todo o território brasileiro. Foram excluídos da análise todos os casos que, apesar de notificados, não apresentavam confirmação diagnóstica ou que continha inconsistências.

Foi utilizado o sistema de informação de dados das notificações de hanseníase, vinculado à Secretaria Municipal de Saúde e ao DATASUS abrangendo o período entre 2009 e 2013. Esse banco de dados é constituído por todos os casos de hanseníase notificados e confirmados de indivíduos residentes em Montes Claros, através da Ficha Individual de Notificação/Investigação de Hanseníase, arquivada no Sistema de Informação de Agravos Notificação (SINAN).

As variáveis do estudo foram divididas em sete categorias de análise: ano; sexo; faixa etária mais acometida pela doença; forma clínica; idade; classificação operacional; avaliação do grau de incapacidades físicas no diagnóstico e esquema terapêutico. Foram realizadas análises exploratórias (descritivas) dos dados, a partir da apuração de frequências simples absolutas e percentuais para as variáveis categóricas e organização dos resultados em tabelas e gráficos. Como os dados são de domínio público, foi dispensada a aprovação pelo comitê de ética em pesquisa.

\section{Resultados e Discussão}

Os resultados demonstraram que de 2009 a 2013, o que representou cinco anos de estudo, foram registrados 225 novos casos de hanseníase em Montes Claros-MG e o ano com maior ocorrência de Hanseníase foi 2009 , com 73 casos registrados correspondendo a $32,44 \%$ do total do período analisado. Nos dois últimos anos o número de casos se manteve estabilizado em 32 , sendo os menores resultados desde 2009.

Em uma pesquisa realizada no período de 2005 a 2009 em Montes Claros, percebeu-se um declínio no número de casos. No ano de 2006 foi registrado o maior número de casos $(74) \mathrm{e}$, em 2008, o menor (45). De modo geral, o Brasil não demonstrou significativo declínio nos últimos anos, como citado anteriormente, permanecendo ainda elevado o número de casos notificados, apesar da alta cobertura da poliquimioterapia. No entanto, o Estado de Minas Gerais apresenta um quadro melhor quando comparado ao do Brasil ${ }^{5}$.

Destes 225 infectados, 126 deles foram referentes a pessoas do sexo masculino, correspondendo a $56,00 \%$ e 99 foram em pessoas do sexo feminino, correspondendo $44 \%$ do total de pessoas diagnosticadas com hanseníase. Em um estudo realizado entre os anos de 2005 a 2009, ou seja, cinco anos de estudo, foram notificados 330 novos casos de hanseníase neste período. Quando comparado ao estudo atual pode ser observado uma redução nos números de casos notificados em Montes Claros. Quanto ao gênero pesquisado, o gênero masculino correspondeu a $54,5 \%$ enquanto o feminino foi de $45,5 \%{ }^{5}$.

O gráfico I mostra o número de casos notificados de hanseníase estratificado por ano e sexo de ocorrência. 
Tabela I. Distribuição dos registros, segundo ano, sexo, idade, forma clínica, classificação operacional, avaliação do grau de incapacidades físicas no diagnóstico e esquema terapêutico entre os casos de hanseníase, Montes Claros/MG, 2009 a 2013.

\begin{tabular}{|c|c|c|}
\hline Variáveis & $\mathbf{n}$ & $\%$ \\
\hline \multicolumn{3}{|l|}{ Ano } \\
\hline 2009 & 73 & $32,44 \%$ \\
\hline 2010 & 40 & $17,78 \%$ \\
\hline 2011 & 48 & $21,33 \%$ \\
\hline 2012 & 32 & $14,22 \%$ \\
\hline 2013 & 32 & $14,22 \%$ \\
\hline \multicolumn{3}{|l|}{ sexo } \\
\hline Masculino & 126 & $56,00 \%$ \\
\hline Feminino & 99 & $44,00 \%$ \\
\hline \multicolumn{3}{|l|}{ Idade } \\
\hline$\leq 19$ anos & 5 & $2,22 \%$ \\
\hline $20-49$ anos & 160 & $71,11 \%$ \\
\hline$\geq 50$ anos & 53 & $23,56 \%$ \\
\hline \multicolumn{3}{|l|}{ Forma clínica } \\
\hline Indeterminada & 14 & $6,22 \%$ \\
\hline Tuberculóide & 11 & $4,89 \%$ \\
\hline Dimorfa & 101 & $44,89 \%$ \\
\hline Virchowiana & 88 & $39,11 \%$ \\
\hline IGN/Branco & 2 & $0,89 \%$ \\
\hline Não Classificada & 4 & $1,78 \%$ \\
\hline \multicolumn{3}{|l|}{ Classificação Ope racional } \\
\hline Paucibacilar & 29 & $12,89 \%$ \\
\hline Multibacilar & 196 & $87,11 \%$ \\
\hline \multicolumn{3}{|l|}{ Grau de Incapacidades } \\
\hline Grau 0 & 171 & $76,00 \%$ \\
\hline Grau 1 & 26 & $11,56 \%$ \\
\hline Grau 2 & 13 & $5,78 \%$ \\
\hline IGN/Branco & 9 & $4,00 \%$ \\
\hline Não Avaliado/ Não Informado & 6 & $2,67 \%$ \\
\hline \multicolumn{3}{|l|}{ Esquema Terapêutica } \\
\hline PQT/PB/6 DOSES & 26 & $11,56 \%$ \\
\hline $\mathrm{PQT} / \mathrm{MB} / 12$ DOSES & 193 & $85,78 \%$ \\
\hline IGN/BRANCO & 3 & $1,33 \%$ \\
\hline OUTROS ESQUEMAS SUBSTITUTIVOS & 1 & $0,44 \%$ \\
\hline TOTAL & 225 & $100,00 \%$ \\
\hline
\end{tabular}

Fonte: SINAN, 2014. Secretaria Municipal de Saúde, Montes Claros/MG, 2013.

Isso confirma o fato de que mulheres, de maneira geral, procuram mais os serviços de saúde e neste caso, tratam mais "precocemente" a hanseníase, evitando incapacidades. Isso seria um fator de busca do tratamento mais rápido, pois as mulheres, de um modo geral, se mostram mais preocupadas com a autoimagem do que os homens. ${ }^{7}$

Quanto à faixa etária mais atingida pela doença observa-se uma concentração nas faixas de 20 a 39 anos e 40 a 59 anos, sendo as últimas as mais atingidas.

Estudo mostra que este é um indicador importante na prevalência das incapacidades, o fato de haver maior número de casos na faixa economicamente ativa, e, portanto, sob o risco de exclusão da cadeia produtiva de adultos e, algumas vezes, de adultos jovens ${ }^{7}$. Contudo, podendo gerar comprometimento da dinâmica econômica familiar, pelos efeitos e acometimento 
que a doença pode causar, ao considerar que a população economicamente ativa é mais afetada ${ }^{8}$.

A Organização Mundial da Saúde, em 1982, para fins terapêuticos classificou a hanseníase, conforme o índice baciloscópico, em paucibacilar (índice baciloscópico< 2+) e multibacilar (índice baciloscópico> ou igual a 2+) e, em 1988, estabeleceu que critérios clínicos, considerando paucibacilares casos com até cinco lesões cutâneas e/ ou um tronco nervoso acometido e multibacilares

Gráfico I. Distribuição dos registros e comparação por sexo entre os casos de hanseníase, Montes Claros/MG, 2009 a 2013.

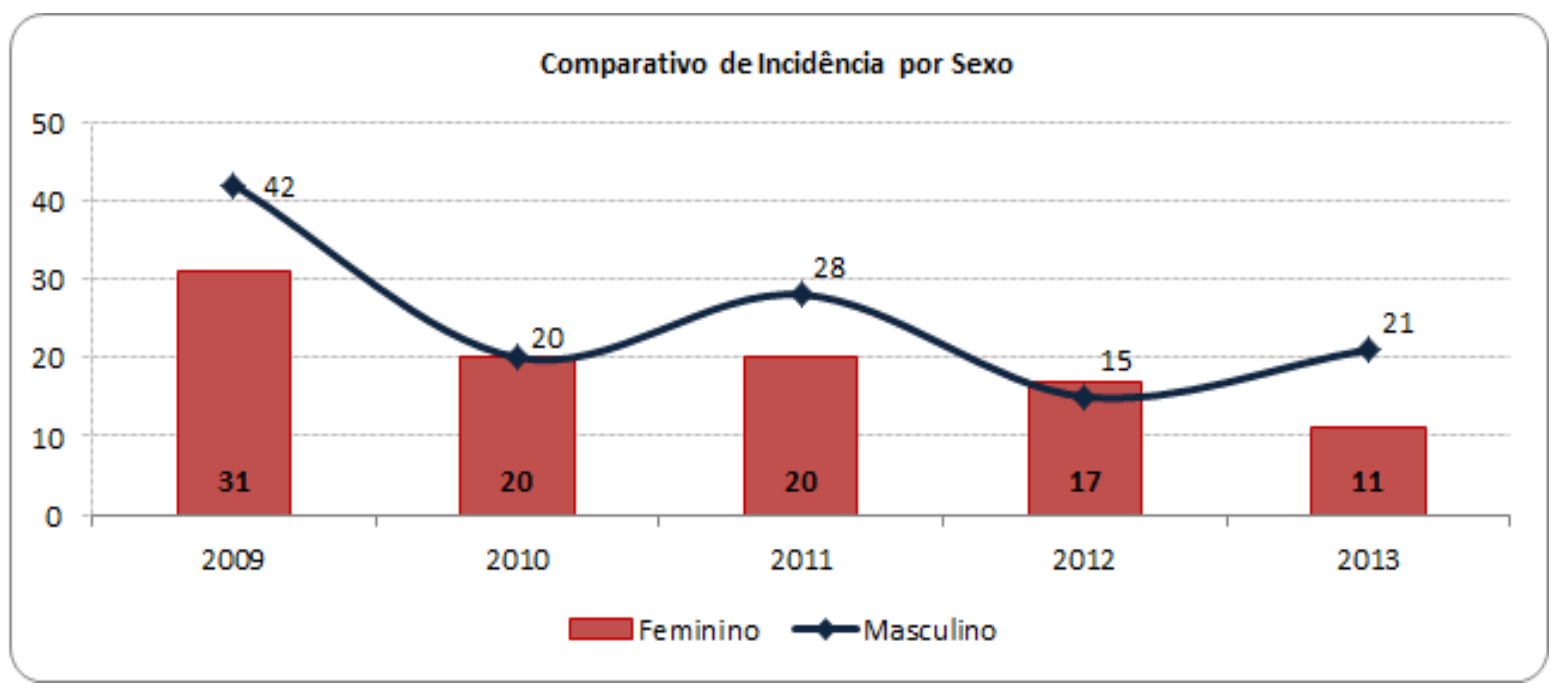

Fonte: SINAN, 2014. Secretaria Municipal de Saúde, Montes Claros/MG.

Gráfico II. Distribuição dos registros e classificação operacional entre os casos de hanseníase, Montes Claros/MG, 2009 a 2013.

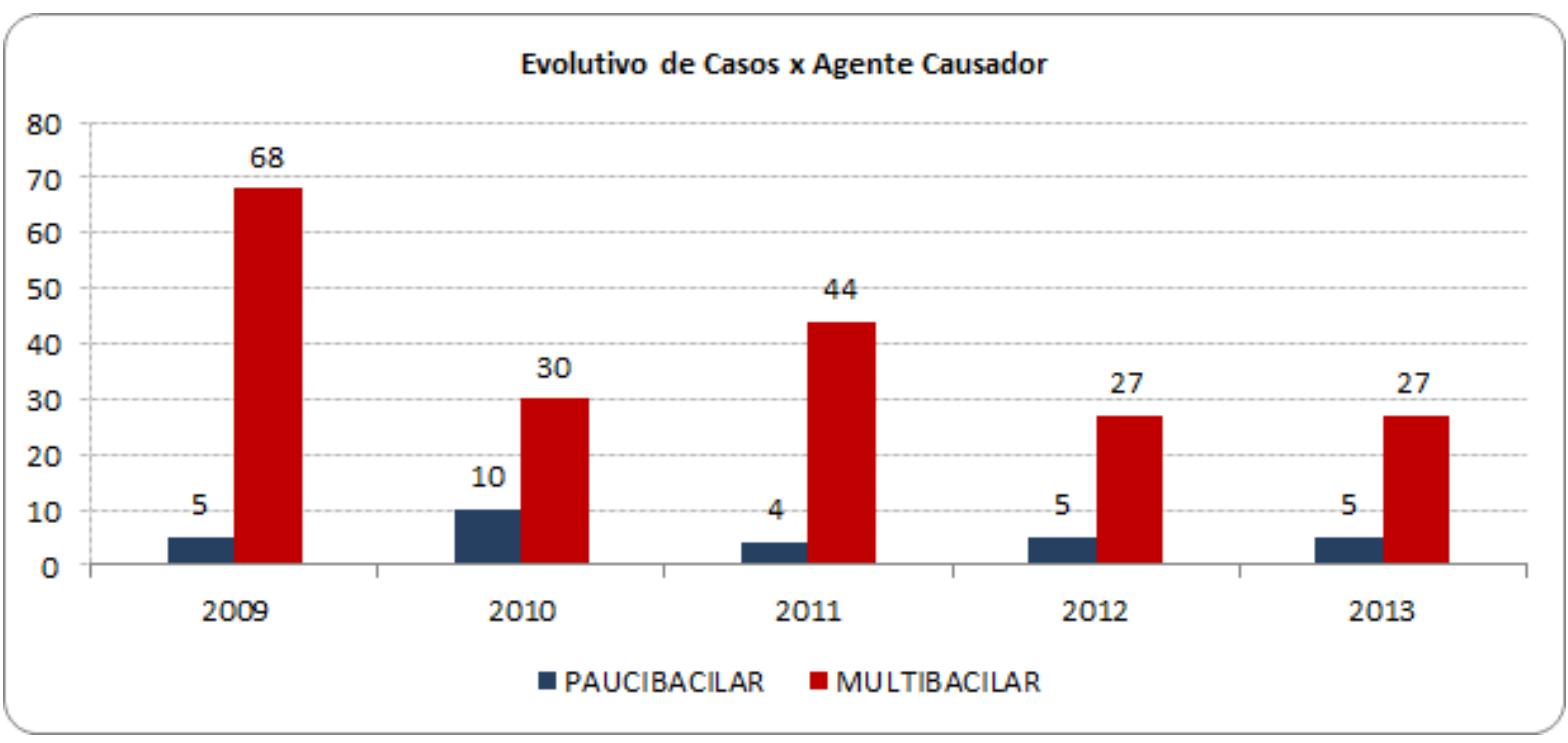

Fonte: SINAN, 2014. Secretaria Municipal de Saúde, Montes Claros/MG, 2013. 
casos com mais de cinco lesões cutâneas e/ou mais de um tronco nervoso acometido. Nos casos em que o exame baciloscópico está disponível, os pacientes com resultado positivo são considerados multibacilares, independentemente, do número de lesões ${ }^{9}$.

A maior parte dos casos de hanseníase pesquisados no período foi causada por agente multibacilar, que correspondeu a $87,11 \%$, constituindo quase o total de casos diagnosticados, enquanto, os casos por agente paucibacilar foram de 12,89\%, isto indica, por meio de diagnóstico tardio, que a população é pouco conscientizada sobre a hanseníase. O sistema de atendimento identifica-se como precário e epidemiologicamente ineficiente de modo que continue o foco de transmissão ou podemos também pensar em uma estabilização da endemia quando os indivíduos mais susceptíveis são afetados, poupando os imunologicamente competentes ${ }^{9}$.

O ano de 2009 foi o ano de maior índice causado por agente multibacilar, correspondendo a $68 \%$ dos casos, seguidos do ano de 2011 que apresentou 44\%, enquanto 2010, registrou uma queda, apresentando o valor de $30 \%$. No entanto, no ano de 2012 a 2013 manteve-se a estabilidade de $27 \%$.

Em contrapartida, o ano de 2010 foi o de maior índice causado por agentes paucibacilar correspondendo a $10 \%$, seguido dos anos de 2009 , 2012 a 2013 apresentando 5\%, enquanto no ano de 2011 foi o de menor índice.

Os tipos mais comuns da doença são a Dimorfa e Virchowiana, nas quais juntas representam a maioria absoluta das ocorrências. Elas são reconhecidas pelo grande poder de transmissibilidade e elevado índice de incapacidade residual. Esses dados devem ser notadamente discutidos entre os profissionais de saúde como estratégia de sensibilização para o diagnóstico e abordagem precoces $^{10}$.

Em relação à forma clínica, observou-se que predominou, na maioria dos usuários, a forma dimorfa $(44,89 \%)$, seguida pela virchowiana $(39,11 \%)$, fato coincidente com outros estudos. Essa situação indica que o diagnóstico tem ocorrido tardiamente, colaborando para a manutenção da cadeia de transmissão. No entanto, este fato seria um sinal de estabilização da endemia ou de ocorrência de baixa prevalência, uma vez que

Gráfico III. Distribuição do histórico da forma clínica entre os casos de hanseníase, Montes Claros/MG, 2009 a 2013.

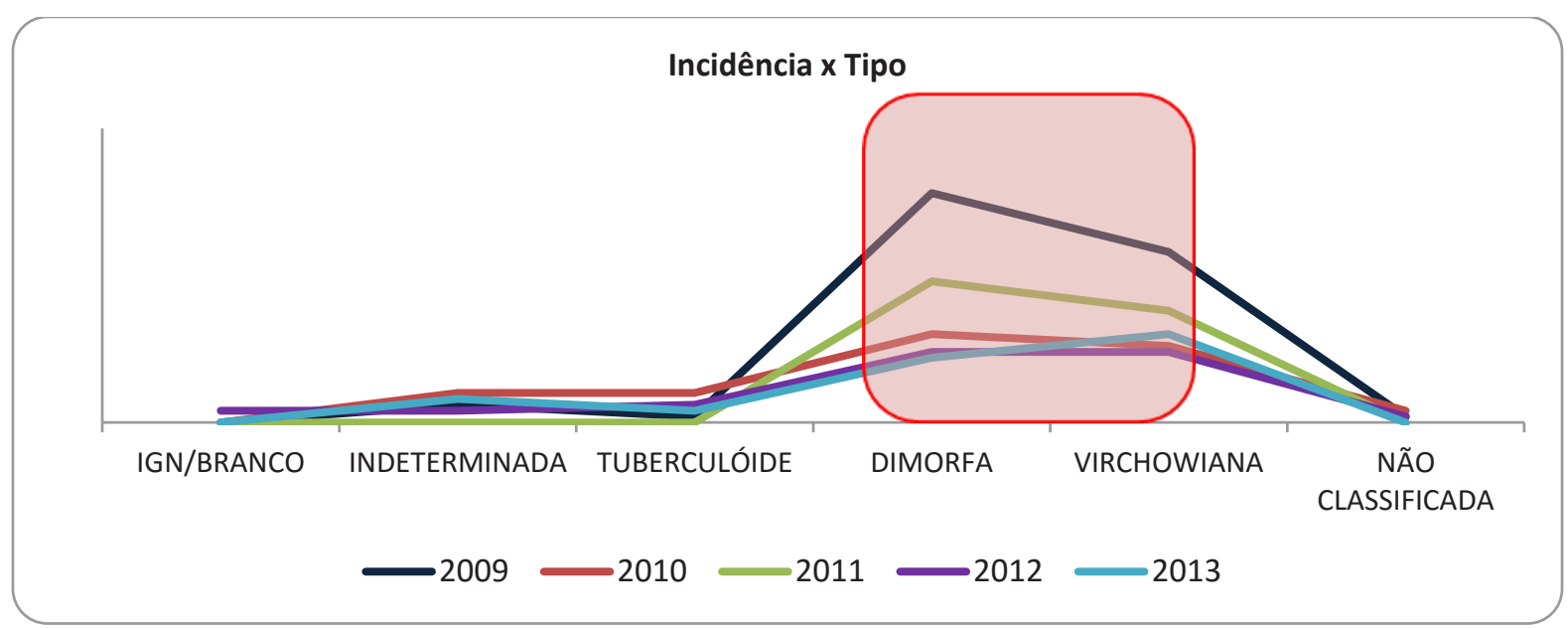

Fonte: SINAN, 2014. Secretaria Municipal de Saúde, Montes Claros/MG, 2013. 
Gráfico IV. Distribuição do histórico de incidência por grau de incapacidade entre os casos de hanseníase, Montes Claros/MG, 2009 a 2013.

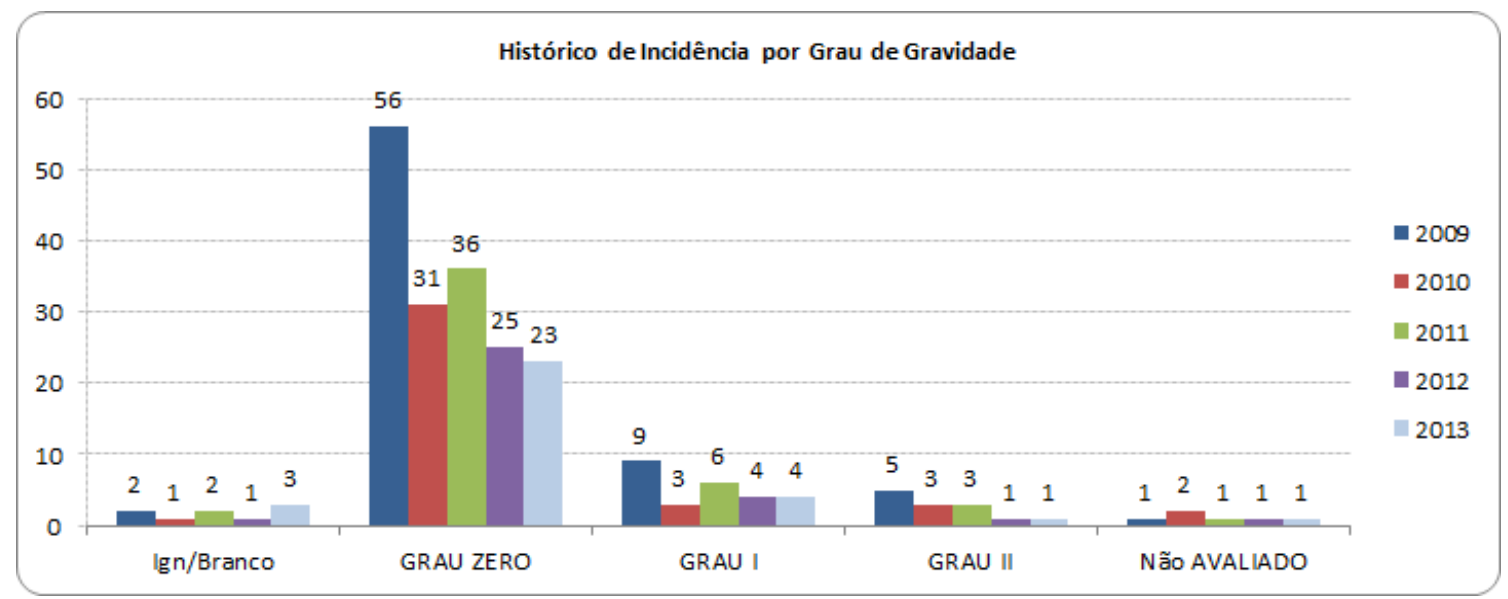

Fonte: SINAN, 2014. Secretaria Municipal de Saúde, Montes Claros/MG, 2013.

apenas os indivíduos mais susceptíveis estariam adoecendo. ${ }^{11}$

É evidente que, ao longo do período, ocorreu um aumento na proporção de casos nas formas multibacilares - dimorfa e virchowiana - enquanto as formas paucibacilares - indeterminada e tuberculóide - decresceram. No total, as formas multibacilares representam a maioria, enquanto as paucibacilares a minoria. Esses resultados podem ser vistos, dentro de um enfoque epidemiológico, como um indicativo de estabilização da endemia ou tendendo a uma situação de baixa prevalência. Por outro lado, o pequeno percentual de casos diagnosticados na forma indeterminada - fase inicial da doença é a desejável para se fazer o diagnóstico. Possivelmente, foram realizados diagnósticos tardios, visto que a evolução para as demais formas, pressupõe, pelo menos dois anos de doença na fase indeterminada ${ }^{12}$.

A maior incidência de tipos de hanseníase no período de 2009 a 2013, quanto ao grau de classificação, é do tipo Grau zero. No período citado ocorreram 171 casos registrados deste tipo da doença, correspondendo a $76 \%$ do total registrado.
Os valores encontrados no presente estudo do nível I devem ser considerados nível baixo (26). Da mesma forma, considera-se baixo o nível de incapacidades do grau II no município (13). No entanto, qualquer proporção de casos diagnosticados com incapacidades tem importância devido ao fato de ser este um indicador de que existe uma prevalência oculta da doença, que, além de gerar tais incapacidades e deformidades, contribui para a manutenção da cadeia de transmissão ${ }^{13}$. Em se tratando da evolução dos casos pesquisados, pode-se observar que existiu um predomínio de cura.

O medicamento mais utilizado no período foi o poliquimioterapia $\mathrm{PQT} / \mathrm{MB} / 12$ DOSES, que corresponde a $85,78 \%$ de tratamento nos casos.

Mesmo sendo de fácil diagnóstico e tratamento, ainda existe dificuldades no controle da hanseníase. Contudo, o esquema de poliquimioterapia (PQT) é o mais recomendado para o tratamento dos doentes, visto que leva a cura, em períodos de tempo considerados curtos, tornando possível desenvolver atividades no controle da doença, mesmo em municípios com menos estrutura ${ }^{14}$. 


\section{Conclusão}

Após a análise dos dados estudados, concluiu-se que o número de casos de hanseníase diagnosticados em Montes Claros-MG apresentou uma redução. A forma multibacilar ainda foi a predominante. Por mais que o número de casos de hanseníase tenha sido reduzido, ainda o agravo é preocupante. A presença de incapacidades indica detecção tardia da doença, tratamento inadequado e conhecimento deficiente, tanto dos profissionais de saúde quanto dos pacientes. A complexidade de enfrentamento de uma doença como a hanseníase leva a refletir e redirecionar os modos de controle da doença, cada vez mais comprometida com a realidade sociossanitária dos territórios onde se pretende intervir.

\section{REFERÊNCIAS}

1) Santos AS, Castro DS, Falqueto A. Fatores de risco para a transmissão da Hanseníase. Revista Brasileira de Enfermagem. 2008; 61(esp): 738-743.

2) Abreu MAMN, Michalany NS, Weckx LLM, Pimentel DRN, Hiratas CHW, Alchorne MMA. A mucosa oral na hanseníase: um estudo clínico e histopatológico. Revista Brasileira de Otorrinolaringologia. 2006; 72(3): 312-316.

3) Ministério da Saúde (BR), Secretaria de Atenção à Saúde. Informe Epidemiológico de Hanseníase, Praia de Iracema - FO. Brasília: Ministério da Saúde, 2013.

4) Ministério da Saúde (BR), Secretaria de Vigilância em Saúde, Departamento de Vigilância Epidemiológica. Hanseníase no Brasil dados e indicadores selecionados. Brasília: Ministério de Saúde, 2009.

5) Santo LRE, Medeiros IA, Cordeiro SS, Teles LF, Silveira MF. Perfil epidemiológico da hanseníase no município de brasileiro no período de 2005 a 2009. Revista Motricidade. 2012; 8 (2): 212-219.

6) Ministério da Saúde (BR), Secretaria de Políticas de Saúde. Guia para o controle da hanseníase. Brasília: Ministério da Saúde, 2002.

7) Pacheco MAB, Aires ML, Seixas ES. Prevalência e controle de hanseníase: pesquisa em uma ocupação urbana de São Luís, Maranhão, Brasil. Revista Brasileira de Medicina de Família e Comunidade. 2014; 30(9): 23-30.

8) Junior AFR, Vieira MA, Caldeiras AP. Perfil epidemiológico da hanseníase em uma cidade endêmica no Norte de Minas Gerais. Revista Brasileira Clínica Médica São Paulo. 2012; 10(4): 272-277.

9) Melão S, Blanco LFO, Mounzer N, Veronezi CCD, Simões PWTA. Perfil epidemiológico dos pacientes com hanseníase no extremo sul de Santa Catarina, no período de 2001 a 2007. Revista da Sociedade Brasileira de Medicina Tropical. 2011; 44(1): 79-84.
10) Miranzi SSC, Pereira LHM, Nunes AA. Perfil epidemiológico da hanseníase em um município brasileiro, no período de 2000 a 2006 . Revista da Sociedade Brasileira de Medicina Tropical. 2010; 43(1): 62-67.

11) Lana FC, Carvalho APM, Davi RFL. Perfil epidemiológico da hanseníase na microrregião de Araçuaí e sua relação com ações de controle. Escola Anna Nery Revista de Enfermagem. 2011; 15(1): 62-67.

12) Lana FC, Amaral EP, Franco MS, Lanza FM. Detecção da hanseníase no Vale do Jequitinhonha - Minas Gerais: redução da tendência epidemiológica ou problemas operacionais para o diagnóstico? Hansenologia Internationalis. 2004; 29(2): 119-123.

13) Junior GM, Castro GG. Perfil epidemiológico da hanseníase no município de Patrocinio - MG. 2009

14) Silva PLN, Chagas RB, Versiani CMC, Macedo LP, Almeida LML, Santos AG, Majuste R. Perfil epidemiológico dos pacientes notificados com hanseníase no norte de Minas Gerais. Revista Eletrônica Gestão e Saúde. 2013 4(3): 896-907.

\section{CORRESPONDÊNCIA}

Luçandra Ramos Espírito Santo

Rua Sebastião Duarte, 91.

Bairro Morada do Sol, CEP: 39401373.

Montes Claros (MG).

E-mail: la_lu_joao@hotmail.com 